\title{
Sistemas comunitarios de satisfacción a necesidades de conectividad en la sociedad de la información: el caso de Argentina
}

\author{
Susana Finquelievich \\ <susana@finquel.com.ar> \\ Daniel Finquelievich \\ $<$ daniel@finquel.com.ar $>$
}

\begin{abstract}
Este articulo se focaliza en la capacidad de las comunidades, mediante las iniciativas y acciones de diversos actores sociales, tanto privados como provenientes de la sociedad civil, para satisfacer sus necesidades con respecto al acceso a las herramientas de la Sociedad de la Información mediante innovaciones sociales y/o tecnológicas, en ausencia o insuficiencia de estrategias estatales. La hipótesis central de este trabajo es que, del mismo modo que Steven Johnson (2001) plantea que las sociedades poseen la posibilidad de autoorganizarse en sistemas emergentes, hallando las mejores soluciones a sus problemas y sus necesidades, al igual que las comunidades participan en las innovaciones socio-técnicas que les conciernen, los emprendimientos que han dado lugar a la conectividad por medio de los cibercafés y las cooperativas serían una forma de autoorganización social para satisfacer una necesidad social no completamente resuelta por el Estado, por las instituciones educativas ni por otras organizaciones comunitarias. Para sostener este argumento, se analizan dos tipos de estrategias en Argentina: una proveniente de pequeños emprendimientos del sector privado, los cibercafés, y otra que se origina en la Sociedad Civil, las Cooperativas Comunitarias de Telecomunicaciones.
\end{abstract}

\section{De la propiedad de los bienes al acceso, de la invención a la co-creación}

Este artículo se focaliza en la capacidad de las comunidades, mediante las iniciativas y acciones de diversos actores sociales, tanto privados como provenientes de la sociedad civil, para satisfacer sus necesidades con respecto al acceso a las herramientas de la Sociedad de la Información mediante innovaciones sociales y/o tecnológicas, aún en ausencia o insuficiencia de estrategias estatales. Los autores sostienen que el Estado, en los países latinoamericanos en general, y en Argentina en particular - sin merma de su papel de estimulador y regulador de los mecanismos de conectividad debe poner el énfasis en la generación de medios productores de bienes y servicios de la Sociedad de la Información, porque el estímulo al consumo de estos bienes está generado por otros agentes sociales.

Para sostener esta hipótesis, se analizan dos tipos de estrategias en Argentina: una proveniente de pequeños emprendimientos del sector privado, los cibercafés, y otra que se origina en la Sociedad Civil, las Cooperativas Comunitarias de Telecomunicaciones.

El estudio de este fenómeno cualitativo de adopción, generación y provisión de acceso a TICs por parte de diversos actores, incluida la sociedad civil, requiere de una revisión al menos en dos aspectos fundamentales:

- La mutación que madura dentro del sistema económico capitalista, referente al pasaje de la propiedad de los bienes al acceso a los mismos (Ver Rifkin, 2000)

- El proceso por el cual se crean las innovaciones y el camino que recorre hacia su adopción por parte de la sociedad, contemplado desde una óptica de co-creación (Ver Tuomi, 2002).

En "La era del Acceso", Rifkin (2000) sostiene que el camino desde un régimen de propiedad de bienes hacia un régimen de acceso, sustentado en garantizar el uso limitado y a corto plazo de los bienes controlados por redes de proveedores, transforma radicalmente nuestras actuales nociones sobre cómo se ejercerá el poder económico en el futuro cercano. Un número creciente de parcelas del comercio están ya ligadas a la mercantilización de una extensa gradación de experiencias culturales en vez de a los bienes y servicios relacionados con la industria tradicional. Entre ellos, el juego, la música, los mundos virtuales del ciberespacio, todo tipo de entretenimiento o de utilización mediada electrónicamente "se convierte rápidamente en el centro de un nuevo hipercapitalismo que comercia con el acceso a las experiencias culturales" (Rifkin, 2000, p.17).

La importancia del acceso sobre la propiedad sugiere que en la Sociedad de la Información, sería relativamente irrelevante la posesión de equipamientos para lograr la conectividad. El cambio se esta dando en la comercialización del tiempo, mas que en la propiedad de los objetos. 
En cuanto a las innovaciones sociotécnicas para la satisfacción de necesidades sociales relativas a la SC, Ilkka Tuomi (2005, in Haddon et. Alt., 2005) se focaliza en la comprensión social del "uso", de un modo menos estrecho y utilitario del que es utilizado por varias empresas de tecnología de informática y telecomunicaciones. Este autor, que se concentra, más que en usuarios individuales, en las relaciones entre los usuarios, en particular comunidades de usuarios, examina las prácticas sociales, registrando de qué maneras las máquinas, los bienes y servicios de base tecnológica son usados socialmente en la gestión de las relaciones entre las personas.

Tuomi (2002) distingue la innovación de la invención. Tradicionalmente, se ha definido a la invención como un proceso de insight creativo y de esfuerzos heroicos para resolver un problema, mientras la innovación era descripta como un proceso que redefine las invenciones y que las traduce en objetos utilizables. En este enfoque clásico, era fácil definir tanto al inventor (sujeto del proceso de invención) y a la invención (objeto): el momento de la invención creaba simultáneamente al inventor y a la invención. Coherentemente con esto, el desarrollo tecnológico fue conceptualizado como compuesto por dos fases cualitativamente diferentes: la invención y su subsiguiente desarrollo como producto (Finquelievich, 2007).

El enfoque propuesto por Tuomi (2002) es otro: sostiene que las "nuevas" tecnologías son activamente interpretadas y apropiadas por actores existentes, en el contexto de sus prácticas existentes. En esta misma línea, Claude Fischer (1992) argumenta que los promotores (productores y diseminadores) de una tecnología no necesariamente saben ni deciden sus usos finales. Ellos detectan las necesidades o problemas que la tecnología puede resolver, pero son los usuarios mismos los que desarrollan nuevos usos, y que deciden finalmente qué usos van a predominar. Puestos ante una tecnología, son los usuarios los que crean nuevos usos.

Por esta razón, la tecnología existe en tanto en que la tecnología es usada. En general, no es posible encontrar un único uso de fondo, estable, que defina absolutamente la naturaleza de un artefacto tecnológico. Desde los artefactos tecnológicos más antiguos: un trozo de sílex, la construcción y conservación del fuego, un martillo, hasta la informática, las telecomunicaciones y la energía nuclear, casi todos los objetos tecnológicos poseen muchos mas usos que el pensado originalmente por sus inventores o descubridores. "La tecnología en uso se refiere al uso de tecnología con sentido. A su vez. El uso con sentido esta enraizado en la práctica social" (Tuomi, 2002). A su vez, la práctica social se basa en el sentido colectivo. Por lo tanto, el uso con sentido de la tecnología es inherentemente social y relacionado con las prácticas sociales.

Ahora bien, ¿Cómo se aplican estos conceptos a una sociedad como la argentina, sacudida por periodos de gravísimas crisis y bonanzas económicas significativas, pero no acompañadas de una eficaz redistribución del ingreso? Según Stiglitz (2002) "El colapso de Argentina (de los años 2001-2002) dio lugar a la suspensión de pagos más grande de la historia". La crisis social siguió a la económica: con un desempleo que pasó de 6\% a más del 18\% en el 2002, se pasó de $16.1 \%$ de población por debajo de la línea de pobreza en 1993 a 30\% en el 2002. La economía argentina ha crecido entre 2003 y 2004 a una tasa anual del 9\%. Pero en un país que en el pasado mostraba un "equilibrio social" alto, similar a los países desarrollados (en 1983 solo ostentaba el $6 \%$ de desempleo en y $12 \%$ de la sociedad en la pobreza), hoy el $26,9 \%$ de la población está por debajo de la línea de pobreza. Según indicó el Instituto Nacional de Estadística y Censos, el nivel de indigencia -medido en los 31 aglomerados urbanos más importantes de la Argentinaquedó en 8,7\%, por debajo del 12,2\% de fines de 2005 (INDEC, 2007).

¿Qué iniciativas de uso con sentido de las TIC se encuentran en este contexto? Steven Johnson (2001) plantea que las sociedades poseen la posibilidad de autoorganizarse en sistemas emergentes ${ }^{1}$ no jerárquicos, encontrando las mejores soluciones a sus problemas y sus necesidades. De acuerdo con este concepto, sostenemos como hipótesis central de este trabajo que, de la misma manera que las comunidades participan en las innovaciones socio-técnicas que les conciernen, también generan formas de autoorganización social para satisfacer necesidades -como la de apropiación de las TIC- no completamente resuelta por el Estado, por las instituciones educativas ni por otras organizaciones comunitarias. Esta hipótesis será ilustrada por medio de in estudio de los emprendimientos que han dado lugar a la conectividad colectiva por medio de los cibercafés ${ }^{2}$ y las cooperativas comunitarias de telecomunicaciones.

\section{Los usos de tic en Argentina}

En lo que se refiere al desarrollo del acceso a la Sociedad de la Información en América Latina, en enero del año 2007, existían en Norteamérica 232 millones de consumidores de Internet; en Asia, 389 millones, y en Europa, 313 millones de usuarios. América Latina cuenta con 89 millones de usuarios, lo que representa la menor cantidad de consumidores de todas las regiones, con una tasa de utilización de Internet de sólo 16 por ciento. Sin embargo, el mercado de Internet de América Latina se expande más rápidamente que la de otras regiones: $391.3 \%$ del 2002 al $2007^{3}$. El crecimiento mundial en el mismo periodo fue del $202.9 \%$.

1 Según Johnson, emergencia es lo que ocurre cuando un sistema de elementos simples se organiza espontáneamente y sin leyes explicitas hasta dar lugar a un comportamiento inteligente.

2 Aún cuando muchos cibercafés han resultado de la iniciativa de grandes empresas telefónicas, el sistema de franquicias que han implementado hace que éstos dependan de pequeñas inversiones de emprendedores privados.

3 Estadísticas Mundiales de Internet 2007, http://www.exitoexportador.com/stats.htm 
Argentina muestra un crecimiento de $150 \%$ por ciento desde el año 2002 al 2006: existen actualmente 13 millones de usuarios de Internet en el país, es decir, personas físicas distintas que se conectan al menos dos veces por semana: una penetración del $34 \%{ }^{4}$. De éstas, el $50 \%$ es de nivel socioeconómico alto y el resto pertenece a los niveles medio y bajo $^{5}$. Si bien el perfil se fue desdibujando desde uno relacionado con una elite a otro más parecido a la población argentina, el ingreso que comenzó en el 2000 todavía está lejos del nivel socioeconómico del país. El hogar sigue siendo el principal lugar de acceso, seguido por los locutorios y cibercafés, que potencian la entrada de nuevos usuarios en los últimos tiempos.

Un estudio de D’Alessio Irol (2006) indica que, con respecto al año 2005, hay más de 2.700 .000 usuarios nuevos. La incorporación de esos "nuevos conectados" es mucho más marcada en el interior del países, donde el crecimiento fue del 47,5\%. En la zona de Capital Federal y Gran Buenos Aires, en tanto, el incremento de usuarios fue del 26,8\%. Por su parte, un estudio de Prince \& Cooke (2006) indicaba que a partir del 2002 los usuarios de Internet muestran un crecimiento superior al 30\% sostenido. Se estimaba que el año 2006 finalizaría con 13 millones de usuarios en Argentina

Las características del usuario de Internet han cambiado desde un perfil inicial más relacionado a una elite, con estudios universitarios y altos conocimientos de informática, y con una significativa mayoría de hombres, al usuario actual, caracterizado por los siguientes rasgos: i) Mayor penetración de niveles medios-bajos, quienes comenzaron su ingreso entre el 2000 y 2001; ii) niveles educacionales que descienden año a año (un 35\% tiene nivel máximo alcanzado de secundario); iii) Migración histórica del lugar de acceso: en los primeros tiempos (en el período previo al 2000) fue el trabajo, luego el hogar y por último la mayor parte del acceso se produjo en los lugares públicos de acceso privado (cibers y locutorios); iv) Equiparación de géneros en Internet: la relación entre mujeres y varones llegó al 50\%, debido principalmente a la masificación de Internet y la aparición de contenidos exclusivos para las mujeres; v) Ingreso de edades extremas: los menores de 18 años representan el $17.7 \%$ de los usuarios actuales. La edad promedio se redujo a 28 años; vi) Ingreso de usuarios con menores conocimientos tecnológicos (Prince \& Cooke, 2005).

Si bien el hogar es el lugar preferido de conexión de los usuarios argentinos en el año 2005 (59.3\%), los cibers ocupan el segundo puesto (46\%), seguidos de lejos por el trabajo (19.4\%), y las instituciones educativas (2.2\%. Esta preferencia se debe a diversas razones; entre las positivas: los bajos costos de los cibers, que rondan $1,50 \$(U) \$ 0,50)$ por hora; la frecuencia del uso de la Banda Ancha en ellos y la velocidad de las conexiones; la no necesidad de encargarse de la vigilancia de virus, spywares, y del mantenimiento de las computadoras. Las razones negativas son el alto costo de la mayoría de servidores de cable o ADSL; la vigilancia creciente hacia el uso del e-mail y de Internet por las empresas hacia sus empleados; y la infrecuente existencia de laboratorios de informática o de telecentros al servicio de los estudiantes en los establecimientos educativos.

Los niveles socio-económico medios, y sobre todo los bajos, hicieron su ingreso a través de cibercafés y locutorios: han hallado en estos lugares el sustituto a la compra de una PC y a la conexión de banda ancha, costosas a partir de la crisis. Los usuarios que sólo se conectan desde lugares privados de acceso público representan el $26 \%$ de la población de Internet. El hogar es la principal ubicación donde los usuarios acceden a Internet, con el $46 \%$.

A continuación se describen las estrategias desarrolladas por dos tipos de organizaciones no estatales (empresas y microemprendimientos del sector privado, y cooperativas que reúnen a diversos actores sociales en sus respectivas comunidades) para avanzar en la inclusión informacional.

\section{Un nuevo espacio social: Los Cibercafes}

Los cibercafés son equipamientos extendidos a nivel mundial, aunque tienden a concentrarse en las grandes ciudades, las localidades en las que el acceso hogareño a Internet aun no es masivo, y los lugares turísticos. Un cibercafé (también llamado cyber, Internet café, PC café, etc.) es típicamente un emprendimiento comercial en el que las personas acceden a Internet por medio de un pago, generalmente equivalente a una fracción de tiempo de una hora o minuto. Un número creciente de cafés ofrecen acceso inalámbrico ilimitado. En muchos cibers también se venden bebidas, papelería, insumos de computación, cigarrillos y golosinas, o se ofrecen servicios como fotocopias y envío y recepción de faxes ${ }^{6}$.

En América Latina y el Caribe se han extendido los llamados Telecentros, financiados por el Estado, por empresas o por organismos internacionales; estos telecentros ofrecen con frecuencia cursos sobre TICs, y otras facilidades para la vida comunitaria. En este trabajo, cuando nos referimos a los lugares privados de acceso público, englobamos en este concepto tanto a los locutorios o franquicias de Telefónica y Telecom, como a los microemprendimientos

4 La carencia de estadísticas accesibles y actualizadas con respecto al uso de los cibercafés en Argentina hace que nuestros datos no estén normalizados al mismo año.

5 Estos datos surgen del Perfil del Usuario de Internet en Argentina realizado por Prince \& Cooke (2006), http://www.princecooke.com/estudios/perfil usuariosinternet 2006.asp

6 Este punto del trabajo recoge algunas de las conclusiones de la investigación de Susana Finquelievich y Alejandro Prince "El (involuntario) rol social de los cibercafés en Argentina". 
independientes. Para comodidad del lector, nos referiremos a este conjunto como a "los cyber".

Los números del mercado argentino de Internet han mostrado a lo largo del período de crisis económico-social de 2001-2003 crecimientos bajos pero sostenidos, que llevaron en el 2004 a crecer el doble que el promedio de dicho período, revelando porcentajes superiores al $27 \%$ en conexiones y $33 \%$ en usuarios ${ }^{7}$. El crecimiento de los cibercafés permitió que el nivel de usuarios siguiera creciendo a pesar de la crisis. Los grupos sociales de ingresos más bajos encontraron la forma de ingresar al mundo de la Web desde lugares donde por $\$ 1$ o $\$ 1,50$ (desde U $\$ 0,30$ a U $\$ 0,50$ ) pueden navegar durante una hora, a alta velocidad y sin tener que comprar una computadora. El caso del uso de los cibers en Argentina es también una nueva manera de reconceptualizar las relaciones de propiedad, uso y nueva economía.

Durante los últimos veinte años, se ha originado en Internet un espacio social de interacciones en red. El espacio social producido por estas comunicaciones reticulares se interpenetra con las redes sociales presenciales, y ambas de modifican mutuamente. En este espacio social, ¿cuál es el rol de los cibercafés? No -siempre- son, como los cafés tradicionales, lugares de encuentro físicos: aunque las personas estén sentadas en incómoda proximidad, la relación no se da entre ellos, sino con las personas que están "al otro lado" de las redes electrónicas, ya sea en otra ciudad, país o continente. Nunes (1999) sugiere que, en vez de servir como lugares de reunión, los cibers funcionan como "espacios de otro lugar", en los cuales el potencial de desplazamiento en el ciberespacio resulta ser un modo de membresía. Lo que se comparte en ellos es el nomadismo electrónico entre lugares geográficamente dispersos, en lo que Manuel Castells llama "el espacio de los flujos".

Esta circulación virtual y (en el caso de los viajeros) transgeográfica explicaría por qué, tanto en Argentina como en el resto del mundo, los cibercafés son lugares donde los diversos grupos socio económicos coexisten. Un relevamiento realizado por el equipo de operadores de calle de la Dirección General de Niñez y Adolescencia del Gobierno de la Ciudad de Buenos Aires (GCBA) ${ }^{8}$ asevera que en el año 2006, los chicos en situación de calle gastaban hasta el $70 \%$ del dinero que ganan vendiendo flores, limpiando ventanillas de coches y otras tareas, en los cibercafés.

Así como la calle es un espacio paradigmático para determinar las condiciones de vida y desarrollo de miles de niños y niñas, hay espacios urbanos marginales donde también se expresa la fragilidad social a través de contextos crónicos de pobreza y exclusión infantil. Uno de ellos es el Barrio Ejército de los Andes, más conocido como Fuerte Apache (por razones de violencia, abuso policial e institucional, delincuencia y tráfico de drogas), localizado al oeste del Gran Buenos Aires. Allí viven 35.000 personas. El 20\% de la población del barrio no tiene empleo ni recibe asistencia social alguna. Casi el 10\% tiene entre 15 y 19 años y se estima que el $20 \%$ de los menores de 14 morirá antes de cumplir los 19 , por cuestiones de violencia o drogas. En el Cibercafé Luna', por ahora único cibercafé del barrio, su propietario calcula que "deben venir alrededor de 100 chicos por día". Y añade: si los mandás a aprender computación, capaz que nunca van. Sin embargo, aquí vienen y aprenden: todo lo que existe está en Internet".

Algunos cafés de la Recoleta (uno de los barrios de mayores ingresos de Buenos Aires) reciben chicos de la calle en los horarios más vacíos, del mismo modo que algunas personas sin techo utilizan los cibers para "recibir" y "visitar" desde sus direcciones electrónicas. De alguna manera, la "clase cibernauta" se superpone, por unos minutos o una hora, a las clases sociales. En los cibers, los hombres de negocios, los intelectuales, los estudiantes, los turistas internacionales, pueden compartir los productos tecnológicos de esta sociedad con cibernautas menos favorecidos, como los niños que habitan la calle, aunque esta coexistencia no está libre de prejuicios de clase, de género o de edad.

No sólo para los grupos económicamente menos favorecidos, sino también para las mujeres, los adultos mayores y los habitantes del interior del país, los cibercafés sirven para despegar a las computadoras y las conexiones a Internet del clásico paradigma de la propiedad y el uso individual del hardware y de las conexiones. Los cibercafés se contraponen a este modelo, dado que no se basan en la propiedad de la tecnología, sino en la compra de tiempo para usarla. Los cibercafés hacen que las personas compartan tecnología en espacios privados de uso público, en vez de en lugares individuales. Al favorecer la presencia virtual en la red -a través de una dirección de e-mail o de Messenger, de la participación en un foro o en un chat-, antes que la presencia física (la propiedad de la computadora), también estimulan un modelo "redcéntrico" de apropiación y utilización de las TIC.

7 Las estadisticas utilizadas en este punto pertenecen a sendos estudios realizados por Prince \& Cooke, en los años 2004 y 2005. 8 El relevamiento se realizó entre septiembre de 2006 a febrero de 2007. Los resultados completos de dicho relevamiento se encuentran en la siguiente página de la Dirección General de Niñez y Adolescencia del Gobierno de la Ciudad de Buenos Aires: http://www.buenosaires.gov.ar/areas/des social/niniez adolescencia/institucional/documentos/relevamiento.php? menu id=19438\#a. La muestra alcanzó 405 casos de niños y niñas sobre más de 600 con los que se estuvo trabajando sistemáticamente. Se cubrieron todos los horarios y zonas de la Ciudad. El 15\% es menor de 12 años. Hay más del doble (64\% vs. $31 \%$ ) de varones que de mujeres, aunque la distancia parece achicarse: cada vez hay más mujeres. En lo que se refiere al tiempo de permanencia en calle, sólo el 7\% de los niños está en la calle desde hace más de 5 años, y el $14 \%$ alternan casa y calle.

9 http://www.clarin.com/diario/2006/12/15/um/m-01328451.htm 
Los cibercafés son también, con frecuencia, el lugar donde se accede a Internet por primera vez, donde el usuario inexperto se vuelve cibernauta. Los cibercafés parecen ser el lugar por excelencia de iniciación en el ciberespacio: el número de personas que aprendió pragmáticamente el uso de Internet en los cibercafés (con frecuencia, guiados por comprensivos dueños o empleados de los mismos) es superior al de quienes se instruyeron en sus hogares, y supera largamente a los que lo aprendieron en sus lugares de trabajo, dejando en un lejano cuarto lugar a las instituciones educativas.

El fenómeno del crecimiento de Internet en Argentina, relacionado en gran parte con el auge de los cibers, ilustra los conceptos de Rifkin y de Nunes: los cibercafés cumplen (involuntariamente) un rol social, dado que facilitan la transición desde un régimen de propiedad de bienes hacia un régimen de acceso de productos y servicios, basado en facilitar el uso limitado y a corto plazo de los bienes controlados por redes de proveedores. Por otro lado, son también, siguiendo a Nunes, los cibercafés son equipamientos que, desde su anclaje territorial, barrial, permiten la movilidad: la movilidad de los usuarios "tecnologizados", no desde dispositivos que lleven consigo, como los teléfonos celulares o las palm tops, sino porque les facilita múltiples puntos fijos de conexión dispersos en el territorio, tanto en su ciudad o país como en el extranjero.

\section{Internet, el mundo de los jóvenes}

Rifkin (2000, Pág. 23) opina que para los jóvenes de principios del siglo XXI, que él llama "la generación proteica", el acceso es una forma de vida, y aunque la propiedad de bienes - como el hardware- continúa siendo importante, lo es más estar conectados: "Las personas del sigo XXI se perciben a sí mismas tanto como nodos insertos en una red de intereses compartidos como agentes autónomos en un mundo darwiniano de supervivencia competitiva. Para ellas la libertad personal tiene menos que ver con el derecho de posesión y la capacidad para excluir a otros, y más con el derecho a estar incluidos en las redes de interrelación. Son la primera generación de la era del acceso".

Los jóvenes son los consumidores más frecuentes de los cyber: los usuarios que tienen hasta 25 años de edad sumaban en 2004 el 48,75 del total relevado. No obstante, en los últimos años se ha registrado la incorporación de edades muy bajas: los menores de 18 años representan el $26 \%$ de los usuarios actuales. Además de actividades muy visibles, como las "de ocio", como Chat y juegos en red, las razones que explica la presencia mayoritaria de jóvenes en los cibers son las siguientes: i) Mayor familiaridad con la tecnología, que ha entrado como una presencia ineludible en sus vidas desde hace unos quince años, convirtiéndose en un ítem de uso irrenunciable; ii) Necesidad de uso de Internet para sus estudios, ya sea para buscar información, interactuar con otros estudiantes, cursar carreras o cursos virtuales, buscar becas, etc.; iii) Aún si algunos de estos jóvenes poseen computadoras en sus casas, las familias pueden limitar el uso de Internet debido a costos telefónicos, uso compartido de computadoras u otras razones; iv) Los cyber se han transformado en lugares donde se pasa el tiempo libre, socializando a través de la red, en soledad, o acompañados por amigos.

La carencia de computadora en el hogar es la primera razón para usar los cibers. Esta tendencia se acentúa aún más entre los que tienen entre 36 y más de 45 años. Estos usuarios también aprecian factores como la comodidad y la facilidad para controlar gastos. Por el contrario, no tener conexión a Internet es una razón para empujar a los cibers a los que tienen entre menos de 18 años hasta los 35 años. Esta franja etaria también privilegia la velocidad, y hasta los 25 años, no estar en su hogar. Los mayores de 45 años usan mayoritariamente la conexión hogareña, doblando al uso de los cibers, salvo cuando están de viaje, tienen problemas con sus conexiones, u otras razones. Sin embargo, en los últimos años se ha incrementado el uso de los cibers por personas mayores. Un grupo de usuarios de Internet que casi se duplicó en el 2002 fue el de las personas mayores de 50 años, alcanzando el $10 \%$ de los usuarios ${ }^{10}$.

La observación directa en cibers de Buenos Aires denota cierta segregación por edad: los jóvenes y los adultos no suelen elegir computadoras cercanas. Inclusive muchos cibers que han optado por un público adulto no han colocado juegos en sus computadoras, para evitar los usuarios más jóvenes y ruidosos, que pueden molestar a los adultos que van a utilizar el e-mail.

En los años post-crisis socio-económica, los cibercafés y las cooperativas comunitarias de telecomunicaciones han provisto a las comunidades de la conectividad que ni el Estado ni los establecimientos educativos les proporcionaban.

\section{Cooperativas comunitarias para la democratización de las comunicaciones}

El caso de las cooperativas comunitarias de telecomunicaciones ilustra un ejemplo de satsfacción de necesidades de al comunidad ejercido por una asociación entre entidades de la sociedad civil y privadas, con en algunos casos, participación del Estado. Para Tuomi, la comunidad crea usos potenciales específicos de la tecnología. Por lo tanto, el "usuario" de una tecnología no es una persona individual, sino un miembro de la comunidad de practica que utiliza la tecnología en cuestión. Si tomamos el caso de una biblioteca, el usuario no es solo cualquier individuo, sino el bibliotecario, o lector habitual que practica el uso de la tecnología en cuestión, que conoce sus códigos y que mediante

10 LaVaca. Org, Cumbre Mundial de la Sociedad de la Información, La brecha digital en la Argentina, http://www.lavaca.org/notas/nota421.shtml, consultada el 5 de marzo de 2005. 
sus demandas y necesidades ha contribuido a que la tecnología se adapte a las necesidades de esta comunidad específica y que a su vez descubre usos diferentes para una tecnología dada. Este concepto puede aplicarse a las cooperativas de telecomunicaciones argentinas.

Una cooperativa de telecomunicaciones, que provee telefonía e Internet, es básicamente una asociación autónoma de individuos, que unen fuerzas para resolver necesidades y aspiraciones comunes, económicas, sociales o culturales, a través de una empresa de propiedad común y administrada democráticamente. En Argentina, las cooperativas comunitarias de telecomunicaciones (CCTs) fueron creadas en los años 1960s por ciudadanos que deseaban llenar el vacío comunicacional creado por la empresa de telefonía del Estado, ENTel, que no podía proveer servicios en las áreas alejadas o de baja densidad poblacional. En 1989, ENTel fue privatizada, pero las cooperativas continuaron funcionando, y se expandieron hacia comunidades que, por su localización geográfica o por su escasa población, no resultaban rentables para las grandes empresas telefónicas privadas.

Hasta la privatización de los servicios telefónicos en 1989, éstos fueron administrados por ENTel, la empresa estatal monopólica, que proveía servicios con preferencia a áreas urbanas rentables, y eludía las complejidades de áreas de bajos recursos, baja densidad poblacional, o remotas. Una regulación especial permitió la creación de cooperativas telefónicas, organizaciones sin fines de lucro calificadas para suministrar servicios de telefonía en sus áreas geográficas, con la condición de que estas áreas fueran declaradas por ENTel como no rentables. En 1960 un decreto estatal autorizó a ENTel a construir conexiones telefónicas para éstas. Las cooperativas retuvieron sus concesiones cuando ENTel fue finalmente privatizada, junto con otras empresas estatales, en 1989. Como todas las empresas cooperativas, las de telefonía están regidas por la legislación que las define como "empresas sociales", para diferenciarlas de las empresas tradicionales, orientadas al mercado

Estas cooperativas se desarrollaron y prosperaron, introduciendo nuevas tecnologías, como Internet, para proveer a sus miembros de nuevos servicios con costos a su alcance. En Argentina, las dos Federaciones de Cooperativas más importantes que existen actualmente son: FECOTEL (Federación de Cooperativas de Telecomunicaciones Ltda.), y FECOSUR (Federación de Cooperativas del Servicio Telefónico de la Zona Sur). Entre las dos, representaban en el año 2004, 350 CCTs. La Federación más grande, FECOTEL, tiene más de 40 años de antigüedad. El sector cooperativo sirve a más de 2.5 millones de argentinos - aproximadamente el 8\% de la población total del país - con 600.000 líneas telefónicas, factura alrededor de US\$100 millones por año, y emplea a 3,500 personas.

Las fuentes de financiación más importantes para las Cooperativas provienen de sus miembros o accionistas, que incluyen empresas locales interesadas en tener servicios eficientes y económicos de telecomunicaciones locales. Mientras algunas cooperativas piden créditos a bancos nacionales o locales, algunos de los informantes clave entrevistados para este trabajo plantean las dificultades enfrentadas por las Cooperativas para obtener préstamos importantes.

FECOTEL (2004) es muy estricta con respecto al comportamiento ético de las Cooperativas. Sus asociados deben "siempre y en todas sus acciones, respetar los principios cooperativos: membresía abierta y voluntaria; control democrático de la organización por sus miembros; participación económica de sus miembros; autonomía e independencia; educación, formación permanente e información; cooperación entre Cooperativas; y compromiso con la comunidad".

\section{Servicios y expansión de las Cooperativas}

El grado en el que las CCTs utilizan las tecnologías de última generación varía de acuerdo a las necesidades específicas de sus comunidades, y de sus posibilidades económicas. Tanto su autonomía, como, en muchos casos, el pequeño tamaño de la organización, las torna flexibles y les permite adaptarse a las nuevas tecnologías a un ritmo relativamente rápido. Muchas cooperativas están usando redes de datos sobre IP y transportan voz sobre IP, aunque las centrales de conmutación digitales seguirán en servicio aún por muchos años ${ }^{11}$. También utilizan IP inalámbrico en áreas de baja densidad poblacional que aún no han sido alcanzadas por las redes de cables de cobre. Algunas Cooperativas usan corDECT, una tecnología inalámbrica de loop local desarrollada por el Indian Institute of Technology. CorDECT fue diseñada para prestar conectividad simultánea de alta calidad en voz y datos con buena relación costo-efectividad, en áreas rurales.

Las CCTs proporcionan a sus comunidades servicios de telefonía e Internet, con conexión dial-up y/o de banda ancha (ADSL o WiFi), así como telefonía sobre IP, con tarifas significativamente menores que las grandes compañías privadas que actúan en Argentina (Telefónica y Telecom). Muchas Cooperativas proveen servicios gratuitos a 1 comunidad, como cursos sobre tecnologías de información y comunicación (TICs), acceso gratuito a Internet para escuelas públicas, bibliotecas e instituciones públicas (cuarteles de bomberos, policía, hospitales, etc.). Según las autoridades del COMFER, (Comité Federal de Radiodifusión), la nueva legislación de radiodifusión propuesta en Argentina permitirá a las Cooperativas proporcionar también televisión por cable, lo que las tornará actores poderosos en el mercado de las telecomunicaciones. Las TV. CCTs están prontas para ofrecer un paquete que incluye TV por cable, telefonía y acceso a Internet por una tarifa mensual de US\$16 (precios de 2004). 


\section{El uso con sentido}

Como se mencionó más arriba, Tuomi (2002) sostiene que los promotores de una tecnología no son los que necesariamente conocen sus usos finales ni deciden sobre ellos. Los creadores de la tecnología, sus productores, detectan las necesidades o problemas que la tecnología puede resolver, pero son los usuarios mismos los que desarrollan nuevos usos, y que deciden finalmente qué usos van a predominar. Si, puestos ante una tecnología, son los usuarios los que crean nuevos usos, entonces, la tecnología existe en tanto en que la tecnología es usada. En general, no es posible encontrar un único uso de fondo, estable, que defina absolutamente la naturaleza de un artefacto tecnológico. Se plantea que las prácticas sociales estructuran y organizan la vida social, y proveen los cimientos para el procesamiento del sentido colectivo. El sentido (lo que luego determinara el uso con sentido de las innovaciones tecnológicas) se produce y reproduce en las comunidades especificas, que a su vez producen y reproducen el sentido (el uso determinado de ciertas tecnologías). El sentido se origina entonces en las actividades prácticas colaborativas. La comunidad que reproduce sentidos o significados específicos es la que reproduce las prácticas relativas a ellos.

Las Cooperativas Comunitarias de Telecomunicaciones están cumpliendo un rol social pionero en los países de América Latina y el Caribe ${ }^{12}$, no sólo en tanto que proveedoras de servicios, sino también de generadoras de empleo, de factores de apropiación comunitaria de las TIC, y de democratización de las comunicaciones. En nuestra investigación hemos identificado las siguientes ventajas de las Cooperativas:

Las CCTs constituyen herramientas potentes para la democratización de las comunicaciones, dado que la comunidad se transforma en propietaria y administradora de su propia infraestructura de telecomunicaciones. El compromiso hacia la comunidad es un factor fundamental para el éxito de las cooperativas. Esta responsabilidad implica esfuerzos continuos para implementar nuevas tecnologías, eficiencia y transparencia gerencial y administrativa, servicios mejores y más baratos que las demás empresas, y servicios superiores a los usuarios.

La creación de CCTs necesita que la comunidad realice contribuciones financieras importantes - esto no se refiere sólo a los usuarios potenciales, sino también y sobre todo a las empresas locales - así como necesita también de créditos blandos de Bancos nacionales o provinciales. Las comunidades de bajos ingresos pueden experimentar dificultades para implementar CCTs, a menos que puedan negociar las inversiones de Bancos o de organizaciones internacionales. Asimismo, la participación activa de "campeones" o líderes locales lúcidos y entusiastas es esencial para el éxito de las iniciativas de creación y funcionamiento de las CCTs, no sólo en las primeras etapas, sino para la sustentabilidad en el largo plazo.

El en caso de las cooperativas, "ser muchos" es mejor. Las cooperativas pueden obtener mejores resultados, tanto cuando negocian con gobiernos, como con empresas privadas, si conforman redes o federaciones nacionales. La construcción de capacidades es esencial para la realización a largo plazo: la formación continua del personal técnico y administrativo de las cooperativas es un factor clave para su éxito. Las Cooperativas pueden proporcionar los medios tecnológicos (acceso, formación) y la información necesaria para que en las comunidades surjan emprendimientos que relativos a la Sociedad de la Información, eventualmente, spin-offs de las mismas

Así como las Cooperativas urgen de la Sociedad Civil (en asociación con otros actores sociales) para proveer conectividad, surgen iniciativas del sector privado, ya sea a nivel micro (emprendimientos individuales) o macro (empresas telefónicas) para proporcionar a la población lugares privados de acceso público a Internet: los cibercafés.

\section{El fordismo de la sociedad de la información}

Las sociedades, según Steven Johnson (2001), poseen la posibilidad de autoorganizarse en sistemas emergentes ${ }^{13}$ no jerárquicos, encontrando las mejores soluciones a sus problemas y sus necesidades. Los microemprendimientos que han dado lugar a la conectividad por medio de los cibers parecería una forma de autoorganización social (involuntaria) para satisfacer una necesidad social no completamente resuelta por el Estado, las organizaciones comunitarias ni los establecimientos educativos.

Sin embargo, sería engañoso reposar (sólo) en este concepto: en el caso de los cibercafés, dado que su implementación se debe fundamentalmente a iniciativas privadas en busca de lucro, no se consideran las necesidades de acceso de la sociedad como un todo. Por esto es que son más numerosos en términos absolutos en las ciudades grandes y medianas, que poseen universidades y en los centros turísticos. Parte de una población de muy bajos recursos, o habitantes de lugares remotos o de baja densidad poblacional continúan excluidos del acceso, excepto que les sea provisto por cooperativas de telecomunicaciones o por organizaciones comunitarias. Tampoco la conectividad es suficiente por sí misma para integrar a la población a la sociedad de la información: se necesita formación de individuos y grupos, agrupamiento de las personas en emprendimientos, instrumentación de las herramientas de la sociedad de la información para el desarrollo humano, entre otros.

12 Bolivia es una excepción: la Federación de Cooperativas Telefónicas de Bolivia, FECOTEL, fue creada en 1987 en Santa Cruz de la Sierra, http://www.comteco.com.bo/cooperativa/FecEstat.doc

13 Según Johnson, emergencia es lo que ocurre cuando un sistema de elementos simples se organiza espontáneamente y sin leyes explícitas hasta dar lugar a un comportamiento inteligente. 
¿Significa esto que es el Estado quien debe asumir la tarea de la implementación y gestión de los cibers, como se ha intentado en los años 1990s en Argentina? Nuestro trabajo tiende a demostrar que no es siempre necesario que el Estado provea equipamientos sociales de conectividad, aunque sí a regular y optimizar el funcionamiento de estos equipamientos privados de uso público.

Regular significa establecer normas de equipamiento (calidad del hardware, por ejemplo), de comodidad de los usuarios, de horarios de uso, de alentar el establecimiento de cibers en regiones de baja densidad poblacional, asegurar que las organizaciones comunitarias puedan establecer acuerdos con los cibers para el uso de la infraestructura con objetivos sociales, etc. Optimizar quiere decir, en este caso, incrementar las funciones de los cibers, incluyendo cursos de formación en uso de TICs, su utilización para fines sociales, etc. De este modo, el Estado se libera de la necesidad de implementar costosas infraestructuras, y al mismo tiempo se asegura de que toda la población esté cubierta por el acceso a Internet y a los servicios asociados a él. Por otro lado, la población, ya familiarizada con el uso cotidiano de los cibers para todo tipo de comunicación, está más dispuesta a avanzar hacia otros usos de las tecnologías, que superan el mero acceso, y sirven para el desarrollo humano, económico y social de las comunidades.

Los datos disponibles sugieren que en los próximos años Internet crecerá a ritmos parecidos a los del 2006, por la dinámica propia de la Ley de Moore y la de Metcalfe ${ }^{14}$. De acuerdo a ambas leyes, es probable que la frecuencia de uso de los cibers aumente debido al creciente interés de los usuarios individuales y grupales por el sistema de comunicaciones en red. Se prevé en los usuarios la continuación de una fuerte tendencia al ingreso a Internet y a las TIC a través de los lugares públicos de acceso privado.

El aumento de los usuarios de cibercafés es un fenómeno típico de la nueva economía, aunque paradójicamente esto se deba a un principio fordista de los dueños de los cibers: apuestan al incremento del número de usuarios, más que al valor alto de la unidad de tiempo utilizada. Los cyber no sólo proveen conectividad a casi la mitad de los cibernautas argentinos: la suministran preferentemente a los grupos socioeconómicos más bajos, a los habitantes del interior, a los jóvenes y a las mujeres. ¿Puede decirse que cumplen un rol social? Si, aunque este rol sea la consecuencia no deliberada de iniciativas económicas en pequeña escala.

El rol involuntariamente social de los cibers - como el papel deliberadamente social de las CCTs- no se limita al acceso de los usuarios al ciberespacio. En América Latina y el Caribe, las investigaciones académicas, los estudios de mercado, las asesorías para decisores políticos, se han enfocado prioritariamente sobre la conectividad: la atención fue puesta sobre cuáles segmentos de la población tiene acceso a Internet o son usuarios de este medio (Finquelievich et al., 2004). La brecha digital ha sido conceptualizada en términos binarios: un individuo o grupo social tiene acceso o no a Internet, usa esta tecnología o no. Por ejemplo, la Cumbre mundial de la Sociedad de la Información, en sus reuniones mundiales de Ginebra (2003) y Túnez (2005) se ha focalizado en que aún existe una "brecha digital" en el mundo, porque los países en desarrollo están lentos en cuanto a la posesión y uso de TIC, y las comunidades pobres no gozan de sus beneficios. Según la CMSI, para cerrar o disminuir esa brecha, debe haber una "solidaridad digital", por la que los países ricos ayuden al mundo en desarrollo a tener mayor acceso a TIC y las personas de todas partes tengan más acceso a la información y el conocimiento.

Este enfoque ultra simplista implica que, cuando en un país sube el número de habitantes conectados - ya sea debido a políticas estatales o, como en el caso argentino, a la iniciativa privada-, se declara prácticamente ganada la batalla contra esta brecha, dado que una proporción significativa de la población utiliza Internet. Este enfoque asume erróneamente que tener acceso a Internet anula o morigera las inequidades presentes y potenciales, consecuencia de la falta de acceso y uso de este medio. Sin embargo, más allá de la conectividad, existen factores que deben ser considerados cuando se debaten los impactos posibles de Internet en las inequidades socio económicas existentes.

Según un trabajo anterior (Finquelievich, 2004), para beneficiarse de las oportunidades de la Sociedad de la Información, los ciudadanos deben estar preparados para las evoluciones económicas, sociales culturales y tecnológicas que transforman el mundo. La e-readiness de los ciudadanos describe el grado de preparación de la sociedad de un país para participar como agentes proactivos en los diversos sectores y niveles de Economía del Conocimiento, y de capitalizar las oportunidades de participación ofrecidas por el nuevo ambiente económico y tecnológico (IAP, 2000). Esta e-readiness necesita, entre otros, de los siguientes elementos:

- Acceso a las infraestructuras de TICs: hardware, software, conectividad, etc.)

- Formación en el uso de TICs (no solo alfabetización Tecnológica, sino también formación en gestión de empresas, organizaciones, etc. usando las TICs).

- Acceso a Internet rápido, gratuito o a bajo costo.

- Información y creatividad para identificar las oportunidades ofrecidas

14 Ley de Moore: La consecuencia directa de esta Ley es que los precios caen al mismo tiempo que las prestaciones crecen: la computadora que hoy vale 3.000 dólares costará la mitad al año siguiente y estará obsoleta en dos años. Esta ley también se ha usado en otros aspectos tecnológicos, como la memoria o el ancho de banda. La ley de Metcalfe expresa que el valor de un sistema de comunicaciones aumenta proporcionalmente al cuadrado del número de usuarios del sistema $\left(N^{2}\right)$. que el número total de personas que pueden enviar y recibir documentos se incrementa. 
- Uso Efectivo de las TICs. Según Gurstein (2003), el Uso Efectivo puede definirse como la capacidad y la oportunidad de integrar exitosamente a las TICs en el cumplimiento de objetivos propios o colaborativos.

Estos factores - todos los cuales son proporcionados por los actuales cibers y Cooperativas en Argentina contribuyen, no solo a la capacidad de los individuos de utilizar una tecnología en forma efectiva y eficiente, sino también a la e-readiness de una sociedad. Tanto los cibercafés como las Cooperativas proveen acceso a las infraestructuras y a Internet, veloz y a bajo costo, formación, acceso a la información, y oportunidades para el uso efectivo de las TICs.

\section{¿Quién se ocupa de la conectividad?}

El éxito de las Cooperativas y de los cibers en Argentina con respecto a la satisfacción de las necesidades de acceso de parte de la población más pobre, de personas del interior del país, de poblaciones de baja densidad y en zonas remotas, y de mujeres, ¿significa acaso que el impulso a la conectividad y a la e-readiness debe dejarse completamente en manos privadas o de la Sociedad Civil organizada? No, o al menos no sin regulaciones. Pero estas iniciativas privadas han ocupado el lugar dejado, o ineficazmente ocupado, por las carencias o deficiencias de políticas estatales tendientes a la conectividad y a sobrepasar la tan comentada "brecha digital". Bassi y Rabadán (2002) relatan la experiencia del proyecto Centros Tecnológicos Comunitarios (CTC), que había instalado aproximadamente 1350 CTCs distribuidos en todo el territorio nacional. A ellos se suman 1745 Bibliotecas Populares, hoy integradas al Proyecto CTC.

Estas autoras reconocen que "Si bien los CTCs instalados son un hecho, el funcionamiento de los mismos según los objetivos planteados, está muy lejos de optimizarse. La mayoría presentan serias irregularidades, y los que funcionan se encuentran subutilizados". Bassi y Rabadán enumeran las principales dificultades detectadas en visitas a 300 CTCs y expresadas por sus coordinadores, clasificadas en dos áreas: aspectos sociales e institucionales (incluyen factores como la ausencia de un criterio de selección de las instituciones responsables acorde a los objetivos comunitarios planteados, la elección de los futuros coordinadores de CTCs por parte de dichas instituciones no respetó el perfil solicitado, ni los mismos fueron instruidos previamente con respecto a las características del proyecto, falta de definiciones e información acerca de los alcances y límites de la autogestión de los CTCs) y aspectos técnicos, como falta de conexión a Internet en aproximadamente el $50 \%$ de los CTCs instalados debido a los altos costos que implica la conexión en las localidades sin servicio de telefonía con descuento (llamado servicio 0610$)^{15}$ o la necesidad de contar con un soporte técnico provisto por el proyecto CTC ante la frecuente desconfiguración de las redes y otras difícultades técnicas. Evidentemente, y por óptimas que hayan sido las intenciones del Estado argentino para vencer la brecha digital e impulsar la conectividad entre los ciudadanos argentinos, las estrategias implementadas no han sido suficientes para ello, y admiten un alto grado de perfeccionamiento.

La sociedad civil no está ausente de la difusión y aún de la provisión de conectividad a la ciudadanía: por el contrario, posee un papel fundamental en la definición de los nuevos tipos de relaciones y construcciones sociales que deberían desarrollarse a partir de la incorporación de las TIC. No se trata solo de una cuestión de estrategias de gobierno y empresas: es fundamental el fortalecimiento de la presencia de las Organizaciones de la sociedad civil (OSC) en la generación y gestión de las estrategias de conectividad, no solo para promover la solidaridad y confianza entre las personas, sino también para incidir en el las estrategias públicas y políticas. Las OSC asumen cierta incidencia política, para influir en las decisiones y los resultados de las políticas públicas. Inciden fundamentalmente en la visibilidad de los problemas y demandas, ya que en general poseen experiencia y capacidad para descubrirlos y hacerlos públicos, en la formulación de soluciones y propuesta de políticas y en el seguimiento de las acciones emprendidas.

La actitud de las OSC hacia la gestión de la conectividad difiere según las organizaciones: algunas consideran que se debe intensificar la relación con lo público y que las propias organizaciones pueden encargarse directamente de ejecutar algunos programas con manejo propio de los recursos y evaluación del Estado (sobre todo en el nivel municipal). Otras organizaciones no están dispuestas a ocuparse de las funciones que le corresponden al Estado y creen que su actividad debe desarrollarse sólo en el ámbito de lo social y en el control de lo público (Finquelievich y Kisilevsky, 2005).

De todas formas, el sector asociativo argentino no está aún en condiciones de proveer de acceso a la tecnología a la mayoría de la población del país: la realidad es que los ciudadanos de menores recursos, que carecen de computadoras o cuyo equipamiento es demasiado antiguo, una gran parte de habitantes del interior del país, y los más jóvenes, se conectan por ahora, en alta proporción, desde los cibers.

\section{¿Cuál sería el rol del estado?}

¿Qué rol cumple el Estado, cuando otros actores sociales van satisfaciendo necesidades de la sociedad en lugar de, o en ocasiones mejor, que el propio estado? Teóricos como Castells $(2006$, p.27) pronuncian la inminencia de las sociedades Red: "Una red es un conjunto de nodos interconectados (...) una red no posee ningún centro, sólo nodos.

15 El 0610 es un servicio telefónico de tarifa reducida para acceso a Internet. El PSI bonifica \$100 mensuales de la linea telefónica, lo que cubre aproximadamente 6 horas diarias de conexión a través de 0610 o en llamadas locales. 
Los nodos pueden tener mayor o menor relevancia para el conjunto de la red (...). Los nodos existen y funcionan exclusivamente como componentes de las redes: la red es la unidad, no el nodo". Según estos conceptos, el Estado puede funcionar como un nodo imprescindible en las acciones multisectisectoriales, es decir, las iniciativas combinadas de sector público, el sector privado y la Sociedad Civil.

El Estado debería entonces desempeñar su función de nodo en las interacciones entre los diversos sectores, en lo que cada uno (marxianamente) aportará según sus capacidades a las soluciones a las necesidades sociales. Este proceso, según los conceptos de Ester Kaufman, supone la producción e intercambio de información a fin de generar conocimiento que a su vez revierta en un mayor valor en servicios y productos. Estos ciclos significan el acceso y participación para todos los integrantes del sistema multisectorial y también para sus destinatarios finales: los consumidores. Esto supone conexiones horizontales dentro de las instituciones y entre instituciones y también entre actores concretos en contextos híbridos.

Para los autores de este trabajo, el rol del Estado (o uno de ellos) es no sólo el de alentar las interacciones multisectoriales, sino también de posibilitarlas e impulsarlas mediante estrategias y acciones concretas, bajo leyes de juego claras y perdurables.

\section{Para terminar}

Este trabajo plantea que las comunidades, mediante las iniciativas y acciones de diversos actores sociales, tanto privados como provenientes de la sociedad civil, pueden organizarse para satisfacer sus necesidades con respecto a la conectividad en la Sociedad de la Información mediante innovaciones sociales y/o tecnológicas, en ausencia o insuficiencia de estrategias estatales.

Los ejemplos presentados sobre cibercafés y cooperativas muestran que los emprendimientos que han dado lugar a la conectividad por medio de los cibercafés y las cooperativas pueden ser considerados una forma de autoorganización social para satisfacer una necesidad de conectividad de las comunidades insuficientemente completamente solucionada por el Estado, por las instituciones educativas ni por otras organizaciones comunitarias.

Estos emprendimientos contribuyen a ciertas formas de innovación social (de manera deliberada las cooperativas, involuntariamente los cibercafés). Ambos tipos de empresas ven el potencial de beneficios (monetarios o sociales) de las tecnologías, y emplean recursos existentes en nuevas maneras. Ambos suman otros actores a los agentes de conectividad tradicionales. Sin embargo, existen diferencias con los sistemas emergentes planteados por Johnson: no conforman redes, aunque posibilitan el acceso a ellas. Aún no crean valor agregado mediante interacciones significativas. Se limitan a ser nodos de provisión de acceso a las infraestructuras de la Sociedad de la Información. ¿Su conformación en redes que se alimentan mutuamente, iniciará la siguiente etapa?

\section{Bibliografía}

Bassi, Roxana y Silvia Rabadán: Centros Tecnológicos Comunitarios: La experiencia argentina, Congreso Apropiación Social de Tecnologías de la Información y la Comunicación en América Latina y el Caribe, Perú, 17 al 24 de marzo del 2002, http://www.links.org.ar/infoteca/ctc-peru.rtf

Bridges.org (2001), "Comparison of E-Readiness Assessment Models, Final draft, v. 2.13, 14 March 2001, http://www.bridges.org/ereadiness/tools.html

Castells, Manuel (2000) “Is the New Economy Socially Sustainable?” http://www.ul.ie/ icse2000/castellsabstract.html

Castells, Manuel (2006): La sociedad red: una visión global, Alianza Editorial, Madrid.

ECLAC (2002): Panorama social de América Latina 2001-2002, http://www.eclac.cl/cgibin/getProd.asp?xml=/publicaciones/xml/4/11254/P11254.xml\&xsl=/dds/tpl/p9f.xsl

Estadisticas mundiales del Internet (Usuarios del Internet y Población por Países y Regiones), 2007, http://www.exitoexportador.com/stats.htm

Evenson, Laura. "Coffeehouse Patrons Get Wired Cybercafes offer e-mail, Web access." San Francisco Chronicle 2 Sep. 1996: E1.

FECOTEL: Testimonio del estatuto social reformado de la federacion de cooperativas de telecomunicaciones de la republica Argentina ltda. (FECOTEL). Capitulo I. denominación, domicilio, duración y objeto. http://www.fecoteldatos.com.ar/estatuto.doc

Finquelievich Daniel (2006): "Nativos al poder”, en: Finquelievich, Susana (Coordinadora) (2007): La innovación ya no es lo que era. Impactos meta-tecnológicos en áreas metropolitanas", Ed. Dunken, Buenos Aires. 
Finquelievich Susana (2005), Desarrollo local en la Sociedad de la Información. Municipios e Internet, La Crujía, Buenos Aires.

Finquelievich, Susana (2003): ICT and sustainable development in Latin America and the Caribbean, in: Stewart Marshall and Wal Taylor, Editores: Proceedings of $5^{\text {th }}$ International IT in Regional Areas Conference 2003, pp. xii-xxv, Central Queensland University, Rockhampton, Queensland, Australia, ISBN 186674423, 2003.

Finquelievich, Susana (Coordinadora) (2007): La innovación ya no es lo que era. Impactos meta-tecnológicos en áreas metropolitanas", Ed. Dunken, Buenos Aires.

Finquelievich, Susana, and Graciela Kisilevsky Community Democratization of Telecommunications Community Cooperatives in Argentina: The Case Of TELPIN, The Journal of Community Informatics, Vol 1, No 3 (2005), http://www.ci-journal.net/index.php/ciej/article/view/241/0

Finquelievich, Susana, Coord. (2000): “¡Ciudadanos, a la Red!” Ed. La Crujía, Buenos Aires.

Finquelievich, Susana, y Graciela Kisilevsky: La sociedad civil en la era digital: redes sociales sustentadas por tic en Argentina, Documento de Trabajo N. 41, Instituto de Investigaciones Gino Germani, Facultad de Ciencias Sociales, Universidad de Buenos Aires, 2005.

Finquelievich, Susana. La sociedad civil en la economía del conocimiento: TICs y desarrollo socio-económico. Buenos Aires: Instituto de Investigaciones Gino Germani, Facultad de Ciencias Sociales, Universidad de Buenos Aires,

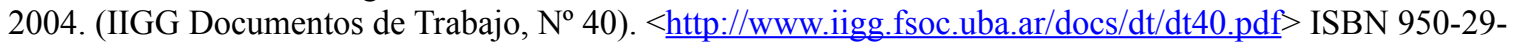
0829-5.

Finquelievich, Susana; Lago Martínez, S.; Jara, A.; Vercelli, A. TIC, desarrollo y reducción de la pobreza: Políticas y propuestas. Buenos Aires: Instituto de Investigaciones Gino Germani, Facultad de Ciencias Sociales, Universidad de Buenos Aires, 2004. (IIGG Documentos de Trabajo, No 37). $<$ http://www.iigg.fsoc.uba.ar/docs/dt/dt37.pdf $>$ ISBN 950-29-0797-3

Gurstein Michael: Community Innovation and Community Informatics Building National Innovation Capability from the Bottom Up, December 2003

Haddon, L, Mante, E., Sapio, B., Kommonen, K-H, Fortunati, L. Kant, A (eds) (2005) Everyday Innovators. Researching the Role of Users in Shaping ICTs, Springer, Dordrect

Instituto Nacional de Estadística y Censos (2007), Calendario de Difusión para el primer y segundo semestre del año 2007, Buenos Aires, http://www.indec.gov.ar/contacto/servicio_calendario.asp

Johnson, Steven: Sistemas emergentes. O qué tienen en común hormigas, neuronas, ciudades y software, Turner Publicaciones, Fondo de Cultura Económica, Madrid, 2001.

Lanvin, Bruno (2001) in The Global Information Technology Report 2001-2002: Readiness for the Networked World, Center for International Development at Harvard University, http://www.cid.harvard.edu/cr/gitrr_030202.html

Obendorf, Tricia (2000): Manuel Castells Leads Keynoters, in Window of the World, Issue 2, http://www.ul.ie/ icse2000/wow/issue2/manuel_castell_keynote.htm

Observatorio de Industrias Culturales del Gobierno de la Ciudad de Buenos Aires, Boletín No 4, "Internet en números. Un mercado que crece", noviembre 2004,

http://www.buenosaires.gov.ar/areas/cultura/observatorio/boletin/gacetilla4.htm, consultado el 5 de marzo de 2004

Prince \& Cooke (2005): PERFIL DEL USUARIO DE INTERNET EN ARGENTINA (NOVIEMBRE 2005), http://www.princecooke.com/estudios/perfil internet_11_2005.asp

Prince \& Cooke (2006): Perfil del usuario de Internet en Argentina 2006 (Diciembre 2006), http://www.princecooke.com/estudios/perfil_usuariosinternet_2006.asp

Rifkin, Jeremy: La era del acceso. La revolución de la nueva economía, Paidós, Buenos Aires, 2000.

Stiglitz, Joseph (2002): Las lecciones de Argentina, Publicado por El País, de Madrid, 10 de enero, 2002, http://www.undp.org.ar/archivos/sobre la crisis de la argentina enero 2002.htm

Stiglitz, Joseph (2003): “Los felices 90”, Taurus, Buenos Aires.

Tapper, Helena: Understanding the Information Society Paradigm, site consulted in 2004, http://www.valt.helsinki.fi/comm/argo/argonet9/TAPPER.HTM

Tuomi, Ilkka (2002): Networks of Innovation. Change and Meaning in the Age of the Internet, Oxford University Press. 